\title{
Demographically-adjusted normative data for the Symbol Digit Modalities Test (SDMT) among Sri Lankan adults
}

\author{
T L Dassanayake, A K A B Baminiwatta, D I Ariyasinghe
}

\section{Abstract \\ Introduction}

The Symbol Digit Modalities Test (SDMT) is a simple pencil-and-paper substitution task that measures divided attention, visual scanning and processing speed. It is sensitive to cognitive impairment in a wide variety of neuropsychiatric conditions. Extant normative data for the SDMT, mainly based on Western populations, may not be applicable for Sri Lankans.

\section{Aims}

We aimed to create sex-, age- and education-adjusted regression-based norms for the SDMT for Sri Lankan adults.

\section{Methods}

Four-hundred and twenty-two community-living adults ( 220 women, $52.1 \%$ ), aged 18 to 83 years, with 5 to 23 years of education, completed the study. We conducted multiple linear regression analyses with sex, age and years of education to predict the SDMT score.

\section{Results}

The SDMT scores of the sample ranged from 5 to 72 , with a mean (SD) score of 35.98 (12.87). The regression model [predicted SDMT score $=29.395-(1.549 \times$ sex $)$ $-(0.434 \times$ age in years $)+(2.207 \times$ years of education $)]$ explained $56.5 \%$ of the variance in SDMT scores (adjusted $\mathrm{R}^{2}=0.565 ; \mathrm{F}=183 ; \mathrm{p}<0.001$ ). Age explained $20.9 \%$ and years of education explained $18.8 \%$ of the SDMT score variance uniquely. Sex was not a significant predictor.

\section{Conclusions}

We report regression-based norms for the SDMT for Sri Lankans aged $18-83$ years, and supplement the regression equation with a Microsoft Excel-based calculator that produces predicted and standardized scores for individual test participants. These norms would assist clinicians in accurately interpreting SDMT test results, accounting for the variability introduced by sex, age and education.

Key words: Symbol Digit Modalities Test; SDMT; regression-based norms; normative data; processing speed

SL J Psychiatry 2021; 12(1): 18-24

\section{Introduction}

The Symbol Digit Modalities Test (SDMT) is a widely used pencil-and-paper-based test of divided attention, visual scanning, and processing speed (1). It is a speeded task that involves substitution of digits against symbols, according to a key displayed at the top of the test sheet. The SDMT was introduced in 1973 by Aaron Smith, and revised in 1982 (2,3). The brevity and ease of administration, unambiguity of scoring criteria, and the availability of both oral and written forms, make it a clinically useful and popular bedside tool in the assessment of patients with neuropsychiatric conditions, and cerebral dysfunction due to other causes $(1,2,4)$.
Group administration of the written form is also possible, and this facilitates its use in community screening (1). An oral form of SDMT is used in evaluating individuals who are unable to make written responses due to physical disabilities or illiteracy.

The SDMT measures similar cognitive abilities as the Digit Symbol Test that appears in Wechsler's intelligence tests (5). Good correlations between SDMT and the Digit Symbol Test performances have been demonstrated (6). As the latter takes the inverse approach of substituting digits with symbols, the more familiar act of responding by writing or speaking numbers makes the SDMT more acceptable to subjects $(1,6)$. 
Despite good sensitivity to cerebral dysfunction in a variety of clinical conditions, the SDMT cannot accurately distinguish between specific disorders (1). For instance, impaired SDMT performance has been seen in organic disorders such as multiple sclerosis (MS), traumatic brain injury, concussion in athletes, Huntington's disease, Parkinson's disease, Alzheimer's dementia and stroke; as well as in psychiatric disorders such as schizophrenia, depression, and attention deficit hyperactive disorder (7-16). The SDMT is part of a standardized batteries for MS, and among their component tests, the SDMT has been shown to be the most strongly associated with neuroimaging indices $(7,17,18)$. Similarly, the SDMT is part of neuropsychological assessment in Huntington's disease, and is one of the few tests sensitive to cognitive deficits in asymptomatic carriers and to the degree of caudate atrophy $(10,19,20)$. Moreover, the SDMT has been shown to be the single best indicator of impaired information processing in traumatic brain injury (8). In schizophrenia, where cognitive deficits are common, the SDMT has shown good test-retest reliability and ecological validity as a measure of processing speed and switching attention $(14,21,22)$. There is some evidence that deficits in all cognitive domains in schizophrenia may be determined primarily by impaired processing speed (23). Depressed individuals, particularly from the older age groups, show poor SDMT performance (15). In organic disorders such as MS, the presence of comorbid depression is associated with greater impairment in SDMT (24).

More importantly, the SDMT has demonstrated sensitivity to deterioration or improvement of cognitive functions, and thus proves useful in monitoring progression of disease and evaluating efficacy of interventions $(25,26)$. SDMT has also shown validity in predicting transition from healthy cognitive aging to mild cognitive impairment (MCI), and from MCI to Alzheimer's dementia, and therefore, is also a valuable tool for early detection of cognitive impairment in the elderly $(27,28)$. The SDMT can be administered from ages 8 to 91 years. Among adults, the test scores decline with advancing age (29). Better educational attainment has also been associated with higher SDMT scores (1,12,30-32). Sex differences have not been evident in many studies but a few have reported females outperforming males $(4,29,33,34)$. Therefore, development of normative data for SDMT should account for the effects of age, education and sex.

Extant normative data for SDMT scores are virtually limited to Western populations, and therefore, may not be applicable in the local setting (4,12,31,32): Even though the written form of the test is relatively free of cultural bias, there is evidence that cultural differences exist for non-verbal tests such as SDMT $(4,35)$. This study is part of a larger project that aims to create demographically-adjusted norms for a number of different neuropsychological tests for Sri Lankan adults $(36,37)$. In this paper, we present sex-, age- and educationadjusted normative data for the SDMT for Sri Lankans, to enable applicability of the test in adults from a wide range of age and educational backgrounds.

\section{Methods}

\section{Study setting and participants}

This study was conducted at the Faculty of Medicine, University of Peradeniya, with the approval of the institutional Ethics Review Committee. Participant recruitment took place from February 2017 to August 2019, through word-of-mouth, flyers and posters displayed within the university and its affiliated tertiarycare hospital - Teaching Hospital Peradeniya. The sample population included employees of the university and the hospital, their acquaintances, and the visitors and caregivers of patients in the hospital. Although data were collected at a single centre, the sample had a wider geographical representation, as the university and hospital employees and the patients referred to the hospital were from various parts of Sri Lanka.

We ensured that an adequate number of participants was included under different age and education categories. Previous normative studies for the SDMT have employed sample sizes ranging from 150 to 3500, and the present sample $(n=422)$ was deemed sufficient to create regression-based norms using three demographic variables (4). The sample had an approximately equal representation of community-living males and females aged 18 years or more. Individuals with a history of serious neurological or psychiatric illnesses, alcohol or other substance dependence, uncorrected visual or hearing impairment, or severe medical illnesses were excluded. Those who were unable to carry out activities of daily living independently were also excluded. However, individuals with other medical conditions that are unlikely to affect their general functioning (e.g. diabetes mellitus) were not excluded: We envisaged such exclusion would cause the sample to be overly healthy thus limiting the generalizability of our norms (1).

The total years of formal education was calculated by summing the years of schooling, graduate and postgraduate education, and post-schooling diplomas. Part-time courses were converted to full-time equivalents. The years of education of the sample varied from 5 to 23 . The sampling did not involve strict stratification based on education or age (which is not essential for regression-based norms), however, we ensured that different educational strata $(\leq 11$ years-Ordinary Level or less; 12-13 years-between Ordinary Level and Advanced Level; 14-17 years-post-schooling diploma, graduate education; $\geq 18$ years-postgraduate education) included participants of different age groups. 


\section{SDMT procedure and outcome measures}

We administered the written form of the SDMT in this study. Instructions were provided verbally. The test form contains a coding key at the top of the page where nine stimulus symbols are paired with the numbers 1 to 9 . The task sequence comprises rows of symbols, each having a blank space underneath, which the subject is instructed to fill consecutively with corresponding numbers as quickly and accurately as possible within 90 seconds. A 10 -item practice trial is provided in the beginning of the first row. The score obtained from the SDMT is the number of correct responses provided within the time limit, with the maximum score being 110 (3).

\section{Statistical analysis}

Preliminary univariate analyses consisted of comparison of mean test scores between males and females (using independent-samples t test), and correlations of the test score with age and years of education. A multiple linear regression (MLR) analysis was performed with sex (coded 0 for females and 1 for males), age in years and years of education as predictors, and the SDMT score as the outcome variable. Data did not violate the assumptions of MLR. Addition of neither quadratic terms nor interaction terms as predictors meaningfully improved the MLR models. We also calculated the semi-partial correlations and shared variances of the SDMT score with sex, age and years of education. The level of significance was set at a cut-off $p$ value of 0.05 . The data were analyzed using IBM SPSS (version 20.0) for Windows.

\section{Results}

\section{Sample characteristics}

The normative sample consisted of 422 participants aged 18.5 to 82.6 years (mean=47.3, $\mathrm{SD}=14.1$ ). The mean $(\mathrm{SD})$ years of education was 13.7 (2.8). The distribution of age, sex and years of education in the sample is summarized in Table 1. The mean age of men (47.28, SD $=13.9$ years) and women (47.34, $\mathrm{SD}=14.4$ years) was similar, $\mathrm{t}(420)=-0.042, \mathrm{p}=0.967$. Also, there was no statistically significant difference between the mean years of education between men (13.7, $\mathrm{SD}=2.6$ years) and women (13.8, $\mathrm{SD}=3.0$ years), $\mathrm{t}(420)=0.033, \mathrm{p}=0.738$. A negative correlation was observed between the age and years of education $(r=-0.296, p<0.001)$, suggesting that the younger generations had received longer education.

\section{SDMT scores and univariate correlations}

The SDMT scores of the sample ranged from 5 to 72 (mean=35.98, SD=12.87). The SDMT scores showed a negative correlation with age $(r=-0.613$, $p<0.001)$, and a positive correlation with the years of education ( $r=0.597, \mathrm{p}<0.001)$ (Figure $1 \mathrm{a}$ and $1 \mathrm{~b})$. The mean scores between women (36.8, SD = 14.31) and men (35.0, SD=11.07) were not significantly different, t (420) $=-1.37, \mathrm{p}=0.172$.

\begin{tabular}{|c|c|c|}
\hline \multicolumn{2}{|c|}{ Table 1. Participant characteristics } & $\%$ \\
\hline Characteristic & Number & 47.9 \\
\hline Sex & & 52.1 \\
Male & 202 & 14.7 \\
Female & 220 & 17.8 \\
Age (years) & & 21.6 \\
$18-29$ & 62 & 23.9 \\
$30-39$ & 75 & 16.1 \\
$40-49$ & 91 & 5.9 \\
$50-59$ & 101 & \\
$60-69$ & 68 & 28.2 \\
$70-83$ & 25 & 23.9 \\
Years of education & & 35.3 \\
$\leq 11$ & 119 & 12.6 \\
$12-13$ & 101 & 149 \\
$14-17$ & 53 & \\
$\geq 18$ & & \\
\hline
\end{tabular}


Table 2. Multiple linear regression model of the predictors of the SDMT score

\begin{tabular}{|l|c|c|c|c|}
\hline & & & \multicolumn{2}{|c|}{ Semi-partial correlations } \\
\cline { 4 - 5 } & B (SE) & $p$ value & $r$ & $r^{2}$ \\
\hline Age in years & $-0.434(0.031)$ & $<0.0001$ & -0.457 & 0.209 \\
Years of education & $2.027(0.150)$ & $<0.0001$ & 0.434 & 0.188 \\
Sex (male=1, female=0) & $-1.549(0.827)$ & 0.062 & -0.060 & 0.004 \\
\hline
\end{tabular}

Note: Adjusted $R^{2}$ for the overall model is $0.565(\mathrm{df}=3,418 ; \mathrm{F}=183 ; \mathrm{p}<0.001)$.

Predicted SDMT score $=29.395-(1.549 \times$ sex $)-(0.434 \times$ age $)+(2.207 \times$ years of education $)$

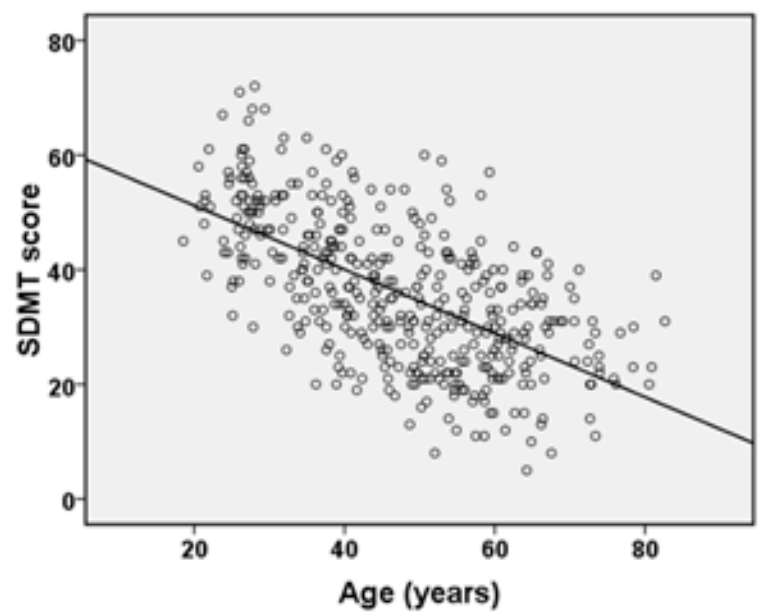

(a)

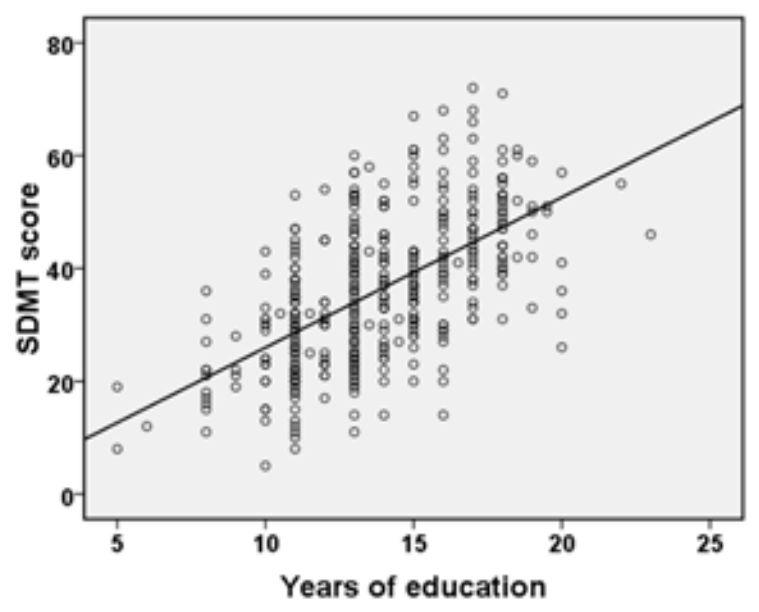

(b)

Figure 1. Relationship of SDMT score with age (a) and years of education (b)

\section{Regression-based norms}

The MLR model that predicted SDMT score based on age, sex and years of education is shown in Table 2. This model showed a good fit, where the three predictor variables explained $56.5 \%$ of the variance in SDMT scores. Age and years of education remained significant predictors of SDMT score in the final model. Semi-partial correlations indicated that age explains $20.9 \%$ and years of education explains $18.8 \%$ of the SDMT score variance. Sex was not a significant predictor and accounted for only $0.4 \%$ of the SDMT score variance. However, we retained sex in the final model to adjust the effect of age and education.

We also present a Microsoft Excel-based calculator, where, upon entering the age (in years), sex ( 0 for females and 1 for males), years of education and the observed raw SDMT score of an individual into the designated cells, the following indicators will be produced: (1) Demographically predicted SDMT score, and (2) The standardized score (i.e. number of standard deviations to the observed score from the predicted score $=$ (observed score - predicted score)/standardized residual). The standardized score enables the clinician to interpret the degree of impairment of a given individual. To provide an example, the predicted SDMT score of a 60-year-old female with 14 years of education is 34.3: if her observed score is 16 , her standardized score is -2.16 : a result that would be interpreted as impaired.

\section{Discussion}

SDMT norms have been published for English-, Dutchand Spanish-speaking populations $(4,31,34,38)$. In the current study, we present demographically-adjusted, regression-based norms based on a community-living sample of Sri Lankan adults aged 18 to 82 years, who had education between 5 and 23 years, enabling the utility of the SDMT in Sri Lanka. It has been repeatedly observed that regression-based norms - compared to traditional demographically-stratified lookup tables - provide greater statistical power and clinical precision for a given sample size $(31,37,39)$. 
The overall mean SDMT score of the present sample (i.e. about 36) is lower than the scores observed in healthy samples from Australia (mean $=49$, age range: $15-100$ years), and the United States (mean scores ranged from 50 to 57 among different age groups from 20-73 years) $(34,40)$. In contrast, mean SDMT scores comparable to present Sri Lankan norms have been reported among Latin American Spanish-speaking populations (e.g. approximately 38 in Mexico, 35 in Chile; 33 in Cuba, 28 in Bolivia and 23 in Paraguay) (38). To illustrate a more accurate, demographically-adjusted comparison using an example, the predicted SDMT score of a 60-year-old female with 14 years of education based on our regression equation (i.e. 34.3), is similar to the predicted scores, if we apply the same demographic data to the regression equations created for the populations from Latin American countries $($ Mexico $=38.8$, Bolivia $=32.8$, and Paraguay $=$ 29.8). In contrast, applying the same demographics into the regression model created for Australians would give a predicted SDMT score of 45, thus falsely pushing the observed performance of the Sri Lankan subject to the subnormal range. These differences highlight the need for country-specific SDMT norms to prevent misclassification of test subjects in clinical settings.

The norms derived from this study can be applied to individuals aged 18 to 82 years. The gradual decline in SDMT performance with advancing age, reported in previous studies was replicated in the present results $(12,29,31,32)$. This may reflect age-related impairment particularly in symbol encoding and visual search (29). Better SDMT performance with longer education is also consistent with previous studies (12,30-32). Since the present sample included individuals with a wide range of education ( 5 to 23 years), these norms can be applied to a population from a broad educational background. In the SDMT manual, education-specific norms are provided, where a dichotomous classification ( $\leq 12$ years versus $>12$ years of education) of educational level is used. We believe, in contrast to the above dichotomized presentation of norms, our regression-based approach - where years of education is modeled as a continuous variable - makes the norms more sensitive to education. For instance, the regression-based approach shows that each 1-year increase in education increases the SDMT score by 2 points - nearly $6 \%$ of the mean SDMT score of the sample. It should be noted that the average years of education in the present sample (13.7 years) is higher than the national average for adults (10.9 years) in Sri Lanka (41). This difference is primarily because we sampled the participants to represent different levels of education (see Table 1). Although this would push the mean SDMT score of the sample higher than the actual population value, the generalizability of the present norms is minimally affected by this since the regression-based approach adjusts for any educational differences.

We did not observe a significant sex difference in SDMT performance, once adjusted for age and education. This is consistent with previous literature, yet there are some exceptions $(4,29,33,34)$. In spite of its minimal contribution to the variation in SDMT scores, we retained sex in the final predictive model, adhering to prior recommendations to use sex-specific norms for cognitive tests $(1,34)$; and the structure of the Sri Lankan norms that we have published previously $(36,37)$.

\section{Limitations}

Although many studies have used education as a predictor of SDMT scores like we did, some others have claimed IQ is a better predictor than education $(18,19,21,23,25)$. It should be noted however, that there are currently no adult IQ tests translated and validated for any of the native languages in Sri Lanka thus precluding any use of IQ as a predictor of specific cognitive test scores. It should be also noted that our norms would be valid only for adults younger than 82 years of age. Whether the same linear decline of SDMT score in our sample stands for those above 82 years is questionable, and therefore, we caution against applying these norms for older individuals. In the same vein, the sample did not include participants with less than 5 years of education whose writing speed rather than cognitive processing could limit the speed of performance in many paper-and-pencil tests. Therefore, the interpretability of SDMT scores of individuals with less than 5 years of education is also contentious.

\section{Conclusions}

Currently, local norms are available only for a handful of neuropsychological tests validated for Sri Lanka (36). In this context, the availability of normative data for the SDMT, a simple, yet powerful test of processing speed, allows clinicians to detect and monitor cognitive impairment in patients with a variety of neuropsychiatric conditions. Finally, the availability of a Microsoft Excelbased digital tool, which calculates the standardized score upon entering the raw score and demographic data, will enable clinicians to easily interpret SDMT scores without the hassle of searching through extensive lookup tables or doing complex hand calculations.

\section{Acknowledgements}

The authors would like to acknowledge the University of Peradeniya for funding this research project through the grant URG/2016/5/M.

\section{Statement of Contribution}

All authors designed the study. TLD and AKABB collected data. All authors analysed data, wrote the manuscript and approved the final version of the manuscript. 


\section{Declaration of Interest}

None declared.

T L Dassanayake, Department of Physiology, Faculty of Medicine, University of Peradeniya, Peradeniya, Sri Lanka and School of Psychology, The University of Newcastle, Australia

A K A B Baminiwatta, Department of Psychiatry, Faculty of Medicine, University of Kelaniya, Sri Lanka

D I Ariyasinghe, Department of Psychiatry, Faculty of Medicine, University of Peradeniya, Sri Lanka

Corresponding author: AKAB Baminiwatta

Email:baminiwatta@kln.ac.lk

http://orcid.org/0000-0002-5495-2029

\section{References}

1. Strauss E, Sherman EMS, Spreen O. A Compendium of Neuropsychological Tests: Administration, Norms, and Commentary. Third Edition. London: Oxford University Press; 2006. 1225p.

2. Smith A. Symbol Digit Modalities Test (Manual). California: Western Psychological Services; 1973.

3. Smith A. Symbol Digit Modalities Test. Manual (Revised). California: Western Psychological Services; 1982.

4. Sheridan LK, Fitzgerald HE, Adams KM, Nigg JT, Martel MM, Puttler LI, et al. Normative Symbol Digit Modalities Test performance in a community-based sample. Arch Clin Neuropsychol Off J Natl Acad Neuropsychol. 2006; 21(1): 23-8.

5. Wechsler D. Manual for the Wechsler Adult Intelligence Scale (WAIS). New York: The Psychological Corporation; 1955.

6. Morgan SF, Wheelock J. Digit Symbol and Symbol Digit Modalities Tests: Are they directly interchangeable? Neuropsychology. US: Educational Publishing Foundation; 1992; 6: 327-30.

7. Christodoulou C, Krupp LB, Liang Z, Huang W, Melville $\mathrm{P}$, Roque C, et al. Cognitive performance and MR markers of cerebral injury in cognitively impaired MS patients. Neurology. 2003; 60(11): 1793-8.

8. Ponsford J, Kinsella G. Attentional deficits following closed-head injury. J Clin Exp Neuropsychol. 1992; 14(5) 822-38.

9. Hinton-Bayre AD, Geffen G, McFarland K. Mild head injury and speed of information processing: a prospective study of professional rugby league players. J Clin Exp Neuropsychol. 1997; 19(2): 275-89.
10. Starkstein SE, Brandt J, Folstein, S, Strauss M, Berthier ML PG et al. Neuropsychological and neuroradiological correlates in Huntington's disease. J Neurol Neurosurg Psychiatry. 1988;51(10):1259-1263.

11. Wills A-MA, Elm JJ, Ye R, Chou KL, Parashos SA, Hauser RA, et al. Cognitive function in 1736 participants in NINDS Exploratory Trials in PD Long-term Study-1. Parkinsonism Relat Disord. 2016; 33:127-33.

12. Fellows RP, Schmitter-Edgecombe M. Symbol Digit Modalities Test: Regression-Based Normative Data and Clinical Utility. Arch Clin Neuropsychol Off J Natl Acad Neuropsychol. 2019; 35(1):105-15.

13. Hsiao PC, Yu WH, Lee SC, Chen MH, Hsieh CL. Responsiveness and predictive validity of the Tablet-based Symbol Digit Modalities Test in patients with stroke. Eur J Phys Rehabil Med. 2019 Feb;55(1):29-34.

14. Chan MWC, Yip JTH, Lee TMC. Differential impairment on measures of attention in patients with paranoid and nonparanoid schizophrenia. J Psychiatr Res. 2004; 38(2): $145-52$.

15. Lyness SA, Eaton EM, Schneider LS. Cognitive Performance in Older and Middle-Aged Depressed Outpatients and Controls. J Gerontol. 1994; 49(3): 12936.

16. Das D, Cherbuin N, Anstey KJ, Easteal S. ADHD Symptoms and Cognitive Abilities in the Midlife Cohort of the PATH Through Life Study. J Atten Disord. 2012 Dec 7; 19(5): 414-24.

17. Beatty WW, Paul RH, Wilbanks SL, Hames KA, Blanco $\mathrm{CR}$, Goodkin DE. Identifying multiple sclerosis patients with mild or global cognitive impairment using the Screening Examination for Cognitive Impairment (SEFCI). Neurology. 1995; 45(4): 718-23.

18. Rao, S.M. A manual for the Brief, Repeatable Battery of Neuropsychological Tests in Multiple Sclerosis. Milwaukee: Medical College of Wisconsin; 1990.

19. Huntington Study Group. Unified Huntington's Disease Rating Scale: reliability and consistency. Mov Disord. 1996; 11(2): 136-42.

20. Lemiere J, Decruyenaere M, Evers-Kiebooms G, Vandenbussche E, Dom R. Longitudinal study evaluating neuropsychological changes in so-called asymptomatic carriers of the Huntington's disease mutation after 1 year. Acta Neurol Scand. 200; 106(3): 131-41.

21. Tang SF, Chen IH, Chiang HY, Wu CT, Hsueh IP, Yu WH, et al. A comparison between the original and Tablet-based Symbol Digit Modalities Test in patients with schizophrenia: Test-retest agreement, random measurement error, practice effect, and ecological validity. Psychiatry Res. 2018; 260: 199-206.

22. Lee P, Li PC, Liu CH, Hsieh CL. Test-retest reliability of two attention tests in schizophrenia. Arch Clin Neuropsychol Off J Natl Acad Neuropsychol. 2011; 26(5): 405-11. 
23. Andersen R, Fagerlund B, Rasmussen H, Ebdrup BH, Aggernaes B, Gade A, et al. The influence of impaired processing speed on cognition in first-episode antipsychotic-naïve schizophrenic patients. Eur Psychiatry. 2013; 28(6): 332-9.

24. Patel VP, Feinstein A. The link between depression and performance on the Symbol Digit Modalities Test: Mechanisms and clinical significance. Mult Scler. 2019; 25(1): 118-21.

25. Koutsouraki E, Kalatha T, Grosi E, Koukoulidis T, Michmizos D. Cognitive decline in Multiple Sclerosis patients. Hell J Nucl Med. 2019; 22(Suppl): 75-81.

26. Morrow SA, O'Connor PW, Polman CH, Goodman AD, Kappos L, Lublin FD, et al. Evaluation of the symbol digit modalities test (SDMT) and MS neuropsychological screening questionnaire (MSNQ) in natalizumab-treated MS patients over 48 weeks. Mult Scler. 2010; 16(11): 1385-92.

27. Cherbuin N, Sachdev P, Anstey KJ. Neuropsychological predictors of transition from healthy cognitive aging to mild cognitive impairment: The PATH through life study. Am J Geriatr psychiatry Off J Am Assoc Geriatr Psychiatry. 2010; 18(8): 723-33.

28. Zhou B, Zhao Q, Kojima S, Ding D, Higashide S, Nagai Y, et al. One-year Outcome of Shanghai Mild Cognitive Impairment Cohort Study. Curr Alzheimer Res. 2019; 16(2): 156-65.

29. Gilmore GC, Royer FL, Gruhn JJ. Age differences in symbol-digit substitution task performance. J Clin Psychology. 1983; 39: 114-24.

30. Richardson ED, Marottoli RA. Education-Specific normative data on common neuropsychological indices for individuals older than 75 Years. Clin Neuropsychol. 1996; 10(4): 375-81.

31. Burggraaff J, Knol DL, Uitdehaag BMJ. Regression-Based Norms for the Symbol Digit Modalities Test in the Dutch Population: Improving Detection of Cognitive Impairment in Multiple Sclerosis. Eur Neurol. 2017; 77(5-6): 246-52.

32. Berrigan LI, Fisk JD, Walker LAS, Wojtowicz M, Rees LM, Freedman MS, et al. Reliability of regression-based normative data for the oral symbol digit modalities test: an evaluation of demographic influences, construct validity, and impairment classification rates in multiple sclerosis samples. Clin Neuropsychol. 2014; 28(2): 281-99.

33. Jorm AF, Anstey KJ, Christensen H, Rodgers B. Gender differences in cognitive abilities: The mediating role of health state and health habits. Intelligence 2004; 32: 7-23.

34. Kiely KM, Butterworth P, Watson N, Wooden M. The Symbol Digit Modalities Test: Normative data from a large nationally representative sample of Australians. Arch Clin Neuropsychol Off J Natl Acad Neuropsychol. 2014; 29(8): 767-75.

35. Fasfous A, Hidalgo N, Vilar-López R, Catena A, PerezGarcia M. Cultural Differences in Neuropsychological Abilities Required to Perform Intelligence Tasks. Arch Clin Neuropsychol. 201; 28(8): 784-90.

36. Dassanayake TL, Ariyasinghe DI. Sex-, age-, and education-adjusted norms for Cambridge Neuropsychological Test Automated Battery in literate Sri Lankan adults. Clin Neuropsychol. 2019; 33(sup1): 106-24.

37. Dassanayake TL, Hewawasam C, Baminiwatta A, Samarasekara N, Ariyasinghe DI. Sex-, age- and educationadjusted norms for the WHO/UCLA version of the Rey Auditory Verbal Learning Test for Sinhala-speaking Sri Lankan adults. Clin Neuropsychol. 2020; 34(sup1): 127-42.

38. Arango-Lasprilla JC, Rivera D, Rodríguez G, Garza MT, Galarza-Del-Angel J, Rodríguez W, et al. Symbol Digit Modalities Test: Normative data for the Latin American Spanish speaking adult population. Neuro Rehabilitation. 2015; 37(4): 625-38.

39. Oosterhuis HEM, van der Ark LA, Sijtsma K. Sample Size Requirements for Traditional and Regression-Based Norms. Assessment. 2016; 23(2): 191-202.

40. Uchiyama CL, D’elia LF, Dellinger AM, Seines OA, Becker JT, Wesch JE, et al. Longitudinal comparison of alternate versions of the symbol digit modalities test: Issues of form comparability and moderating demographic variables. Clin Neuropsychol. 1994; 8(2): 209-18.

41. Human Development Reports. [Internet]. New York: United Nations Development Programme; [updated 2020]. Human Development Indices and Indicators: 2018 Statistical Update [updated 2019] [cited 05.05.2021]. Available from: http://hdr.undp.org/sites/default/files/ 2018_human_development_statistical_update.pdf 